\title{
Self-concordance is NP-hard
}

\author{
Lek-Heng $\operatorname{Lim}^{1}$
}

Received: 15 April 2016 / Accepted: 14 September 2016 / Published online: 19 September 2016

C Springer Science+Business Media New York 2016

\begin{abstract}
We show that deciding whether a convex function is self-concordant is in general an intractable problem.
\end{abstract}

Keywords Self-concordance $\cdot$ Second-order self-concordance $\cdot$ NP-hard $\cdot$ Co-NP-hard

\section{Introduction}

Nesterov and Nemirovskii [15] famously showed that the optimal solution of a conic programming problem can be computed to $\varepsilon$-accuracy in polynomial time if the cone has a self-concordant barrier function whose gradient and Hessian are both computable in polynomial time. Their work established self-concordance as a singularly important notion in modern optimization theory.

We show in this article that deciding whether a convex function is self-concordant at a point is nonetheless an NP-hard problem. In fact we will prove that deciding the self-concordance of a convex function defined locally by a cubic polynomial (which cannot be convex on all of $\mathbb{R}^{n}$ ), arguably the simplest non-trivial instance, is already an NP-hard problem. In addition to the NP-hardness of self-concordance, we will see that, unless $P=N P$, there is no fully polynomial time approximation scheme for the optimal self-concordant parameter and that deciding second-order self-concordance [10] of a quartic polynomial is also an NP-hard problem.

These hardness results are intended only to add to our understanding of self-concordance. They do not in anyway detract from the usefulness of the notion since in practice self-

This work is supported by AFOSR FA9550-13-1-0133, DARPA D15AP00109, NSF IIS 1546413, DMS 1209136, and DMS 1057064.

Lek-Heng Lim

lekheng@galton.uchicago.edu

1 Computational and Applied Mathematics Initiative, Department of Statistics, University of Chicago, Chicago, IL 60637, USA 
concordant barriers are constructed at the outset to have the requisite property (see [15, Chapter 5] and [3, Section 9.6]). We deduce the NP-hardness of self-concordance using a well-known result of Nesterov himself, namely, minimizing a cubic form over a sphere is in general NP-hard [14].

\section{Self-concordance in terms of tensors}

Let $\Omega \subseteq \mathbb{R}^{n}$ be open and $f: \Omega \rightarrow \mathbb{R}$ be in $C^{d}(\Omega)$, i.e., has continuous partials up to at least order $d$. Recall that the $d$ th order derivative of $f$ at $x \in \Omega$, denoted $\nabla^{d} f(x)$, is a tensor of order $d$ [11]. To be more precise, this simply means that $\nabla^{d} f(x)$ is a multilinear functional on $T_{x}(\Omega)$, the tangent space of $\Omega$ at $x$, that is,

$$
\nabla^{d} f(x): \underbrace{T_{x}(\Omega) \times \cdots \times T_{x}(\Omega)}_{d \text { copies }} \rightarrow \mathbb{R},
$$

where

$$
\begin{aligned}
& \nabla^{d} f(x)\left(h_{1}, \ldots, \alpha h_{i}+\beta h_{i}^{\prime}, \ldots, h_{n}\right) \\
& \quad=\alpha \nabla^{d} f(x)\left(h_{1}, \ldots, h_{i}, \ldots, h_{n}\right)+\beta \nabla^{d} f(x)\left(h_{1}, \ldots, h_{i}^{\prime}, \ldots, h_{n}\right) \quad \text { for } i=1, \ldots, n .
\end{aligned}
$$

With respect to the standard basis $\frac{\partial}{\partial x_{1}}, \ldots, \frac{\partial}{\partial x_{n}}$ of $T_{x}(\Omega)$, we may identify $T_{x}(\Omega) \cong \mathbb{R}^{n}$ and $\nabla^{d} f(x)$ may be regarded as a ' $d$-dimensional matrix' or $d$-hypermatrix,

$$
\nabla^{d} f(x)=\left[a_{i_{1} \cdots i_{d}}\right]_{i_{1}, \ldots, i_{d}=1}^{n} \in \mathbb{R}^{n \times \cdots \times n} .
$$

Indeed, we must have

$$
a_{i_{1} \cdots i_{d}}=\frac{\partial^{d} f(x)}{\partial x_{i_{1}} \cdots \partial x_{i_{d}}},
$$

and since $f \in C^{d}(\Omega)$, we get that $a_{i_{1} \cdots i_{d}}=a_{i_{\pi(1)} \cdots i_{\pi(d)}}$ for all permutations $\pi$ on the indices, i.e., $\nabla^{d} f(x)$ is a symmetric $d$-hypermatrix. Every symmetric $d$-hypermatrix $A=$ $\left[a_{i_{1} \cdots i_{d}}\right]_{i_{1}, \ldots, i_{d}=1}^{n} \in \mathbb{R}^{n \times \cdots \times n}$ defines a homogeneous polynomial of degree $d$, denoted

$$
A(h, \ldots, h):=\sum_{i_{1}, \ldots, i_{d}=1}^{n} a_{i_{1} \cdots i_{d}} h_{i_{1}} \cdots h_{i_{d}} \in \mathbb{R}\left[h_{1}, \ldots, h_{n}\right]_{d} .
$$

$d$-hypermatrices are coordinate representations of $d$-tensors, just as matrices are coordinate representations of linear operators and bilinear forms (both are 2-tensors). We refer the reader to [12] for more information.

The usual definition of self-concordance requires that $f \in C^{3}(\Omega)$ and in which case it is given by a condition involving the matrix $\nabla^{2} f(x) \in \mathbb{R}^{n \times n}$ and the 3-hypermatrix $\nabla^{3} f(x) \in \mathbb{R}^{n \times n \times n}$.

Definition 1 (Nesterov-Nemirovskii) Let $\Omega \subseteq \mathbb{R}^{n}$ be a convex open set. Then $f: \Omega \rightarrow \mathbb{R}$ is said to be self-concordant with parameter $\sigma>0$ at $x \in \Omega$ if

$$
\nabla^{2} f(x)(h, h) \geq 0
$$

and

$$
\left[\nabla^{3} f(x)(h, h, h)\right]^{2} \leq 4 \sigma\left[\nabla^{2} f(x)(h, h)\right]^{3}
$$

for all $h \in \mathbb{R}^{n} ; f$ is self-concordant on $\Omega$ if (1) and (2) hold for all $x \in \Omega$. The set of self-concordant functions on $\Omega$ with parameter $\sigma$ is denoted by $S_{\sigma}(\Omega)$. 
By (1), a function self-concordant on $\Omega$ is necessarily convex on $\Omega$. A minor deviation from [15] is that $\sigma$ above is really the reciprocal of the self-concordance parameter as defined in [15, Definition 2.1.1]. Our hardness results would be independent of the choice of $\sigma$. Note that

$$
\nabla^{2} f(x)(h, h)=\sum_{i, j=1}^{n} \frac{\partial^{2} f(x)}{\partial x_{i} \partial x_{j}} h_{i} h_{j}, \quad \nabla^{3} f(x)(h, h, h)=\sum_{i, j, k=1}^{n} \frac{\partial^{3} f(x)}{\partial x_{i} \partial x_{j} \partial x_{k}} h_{i} h_{j} h_{k} .
$$

So for a fixed $x \in \Omega, \nabla^{2} f(x)(h, h)$ is a quadratic form in $h$ and $\nabla^{3} f(x)(h, h, h)$ is a cubic form in $h$. It is well-known that deciding (1) at any fixed $x$ is a polynomial-time problem (but not so for deciding it over all $x \in \Omega$, see [1]). Hence given a $\sigma>0$, deciding selfconcordance at $x$ essentially boils down to (2): Is the square of a given cubic form globally bounded above by the cube of a given quadratic form? We shall see in the next sections that this decision problem is NP-hard.

While we will think of $\nabla^{2} f(x)$ as a matrix and $\nabla^{3} f(x)$ as a hypermatrix, we wish to highlight that (2) is really a condition on $\nabla^{2} f(x)$ regarded as a 2-tensor and $\nabla^{3} f(x)$ regarded as a 3-tensor, i.e., (2) is independent of the choice of coordinates, a property that follows from the affine invariance of self-concordance [15, Proposition 2.1.1]. Self-concordance on $\Omega$ is therefore a global condition about the tensor fields $\nabla^{3} f$ and $\nabla^{2} f$.

\section{Maximizing a cubic form over a sphere}

We include a proof that the clique and stability numbers of a graph with $n$ vertices and $m$ edges may be expressed as the maximum values of cubic forms (in $n+m$ variables) over the unit sphere $\mathbb{S}^{n+m-1}$. This, or at least the stability number version, is known but the reference usually cited [14, Theorem 4] contains some typos that have been reproduced elsewhere ${ }^{1}$. We take the opportunity to provide a corrected version below. Our proof follows Motzkin-Straus Theorem [13] and the similar result of Nesterov [14, Theorem 4] for stability number.

Let $G=(V, E)$ be an undirected graph with $n$ vertices and $m$ edges. We shall require that $E \neq \varnothing$ throughout, so $n \geq 2$ and $m \geq 1$. Recall that $S \subseteq V$ is a clique in $G$ if $\{i, j\} \in E$ for all $i, j \in S$ and $S \subseteq V$ is stable in $G$ if $\{i, j\} \notin E$ for all $i, j \in S$. The clique number and stability number of $G$ are respectively:

$$
\omega(G)=\max \{|S|: S \subseteq V \text { is clique }\}, \quad \alpha(G)=\max \{|S|: S \subseteq V \text { is stable }\} .
$$

Motzkin and Straus [13] showed that $\omega(G)$ may be expressed in terms of the maximum value of a simple quadratic polynomial over the unit simplex. Although not in [13], it is straightforward to see that essentially the same proof also yields a similar expression for $\alpha(G)$.

Theorem 1 (Motzkin-Straus) Let $\Delta^{n}=\left\{x \in \mathbb{R}^{n}: x_{1}+\cdots+x_{n}=1, x_{i} \geq 0\right\}$ denote the unit simplex in $\mathbb{R}^{n}$. Then the clique number $\omega(G)$ and stability number $\alpha(G)$ may be determined via quadratic optimization over simplices:

$$
1-\frac{1}{\omega(G)}=2 \max _{x \in \Delta^{n}} \sum_{\{i, j\} \in E} x_{i} x_{j}, \quad 1-\frac{1}{\alpha(G)}=2 \max _{x \in \Delta^{n}} \sum_{\{i, j\} \notin E} x_{i} x_{j} .
$$

1 For example, [4, Theorem 3.4]. To see that the expression is incorrect, take a graph with three vertices and one edge, the left-hand side gives $1 / \sqrt{2}$ and the right-hand side gives 1 . 
Since deciding if a clique of a given size exists is an NP-complete problem [9], an immediate consequence is that computing the clique number of a given graph is NP-hard, and by the Motzkin-Straus theorem so is the maximization of a quadratic form on a simplex.

In an unpublished manuscript [14, Theorem 4], Nesterov used Motzkin-Straus Theorem to obtain an alternate expression (5) for stability number involving the maximum value of a cubic form over a sphere. In the following we derive a similar expression (4) for the clique number, which yields slightly simpler expressions for our discussions in Sects. 4 and 6, and may perhaps be of independent interest.

Theorem 2 (Nesterov) Let $G=(V, E)$ be an undirected graph with $n$ vertices and $m$ edges. Let $\mathbb{S}^{d-1}=\left\{x \in \mathbb{R}^{d}:\|x\|=1\right\}$ denote the unit $\ell^{2}$-sphere in $\mathbb{R}^{d}$. The clique number $\omega(G)$ and stability number $\alpha(G)$ may be determined via cubic optimization over spheres:

$$
\begin{aligned}
& 1-\frac{1}{\omega(G)}=\frac{27}{2} \max _{(v, w) \in \mathbb{S}^{n+m-1}}\left[\sum_{\{i, j\} \in E} v_{i} v_{j} w_{i j}\right]^{2}, \\
& 1-\frac{1}{\alpha(G)}=\frac{27}{2} \max _{(v, w) \in \mathbb{S}^{n+m-1}}\left[\sum_{\{i, j\} \notin E} v_{i} v_{j} w_{i j}\right]^{2} .
\end{aligned}
$$

Proof This follows from Motzkin-Straus Theorem and the equalities

$$
\begin{aligned}
\max _{x \in \Delta^{n}} \sum_{\{i, j\} \in E} x_{i} x_{j} & =\max _{v \in \mathbb{S}^{n-1}} \sum_{\{i, j\} \in E} v_{i}^{2} v_{j}^{2} \\
& =\max _{v \in \mathbb{S}^{n-1}, w \in \mathbb{S}^{m-1}}\left[\sum_{\{i, j\} \in E} v_{i} v_{j} w_{i j}\right]^{2} \\
& =\frac{27}{4} \max _{(v, w) \in \mathbb{S}^{n+m-1}}\left[\sum_{\{i, j\} \in E} v_{i} v_{j} w_{i j}\right]^{2} .
\end{aligned}
$$

(6) comes from substituting $x_{i}=v_{i}^{2}, i=1, \ldots, n$. As for (7), Cauchy-Schwarz yields

$$
\sum_{\{i, j\} \in E} v_{i} v_{j} w_{i j} \leq\left[\sum_{\{i, j\} \in E} v_{i}^{2} v_{j}^{2}\right]^{\frac{1}{2}}\left[\sum_{\{i, j\} \in E} w_{i j}^{2}\right]^{\frac{1}{2}}
$$

and so

$$
\begin{aligned}
\max _{\|v\|=\|w\|=1} \sum_{\{i, j\} \in E} v_{i} v_{j} w_{i j} & \leq \max _{\|v\|=1}\left[\sum_{\{i, j\} \in E} v_{i}^{2} v_{j}^{2}\right]^{\frac{1}{2}} \max _{\|w\|=1}\left[\sum_{\{i, j\} \in E} w_{i j}^{2}\right]^{\frac{1}{2}} \\
& =\max _{\|v\|=1}\left[\sum_{\{i, j\} \in E} v_{i}^{2} v_{j}^{2}\right]^{\frac{1}{2}}=: \alpha .
\end{aligned}
$$

Let the maximum value $\alpha$ be attained at $\bar{v} \in \mathbb{S}^{n-1}$. We set $\bar{w}_{i j}=\bar{v}_{i} \bar{v}_{j} / \alpha$ for all $\{i, j\} \in E$ (note that $\alpha>0$ if $E \neq \varnothing$ ). Observe that

$$
\sum_{\{i, j\} \in E} \bar{v}_{i} \bar{v}_{j} \bar{w}_{i j}=\frac{1}{\alpha} \sum_{\{i, j\} \in E} \bar{v}_{i}^{2} \bar{v}_{j}^{2}=\alpha,
$$

and $\bar{w} \in \mathbb{S}^{m-1}$ since

$$
\sum_{\{i, j\} \in E} \bar{w}_{i j}^{2}=\frac{1}{\alpha^{2}} \sum_{\{i, j\} \in E} \bar{v}_{i}^{2} \bar{v}_{j}^{2}=1 .
$$


Hence equality is attained in (9) and we have (7). We deduce (8) from

$$
\begin{aligned}
\max _{\|(v, w)\|=1} \sum_{\{i, j\} \in E} v_{i} v_{j} w_{i j} & =\max _{\|v\|^{2}+\|w\|^{2}=1} \sum_{\{i, j\} \in E} v_{i} v_{j} w_{i j} \\
& =\sup _{\beta \in(0,1)}\left[\max _{\|v\|^{2}=\beta,\|w\|^{2}=1-\beta} \sum_{\{i, j\} \in E} v_{i} v_{j} w_{i j}\right] \\
& =\sup _{\beta \in(0,1)}\left[\max _{\|v / \sqrt{\beta}\|^{2}=1,\|w / \sqrt{1-\beta}\|^{2}=1} \sum_{\{i, j\} \in E}\right. \\
& =\left[\max _{\|v\|^{2}=1,\|w\|^{2}=1} \sum_{\{i, j\} \in E} v_{i} v_{j} w_{i j}\right] \times \sup _{\beta \in(0,1)} \beta \sqrt{1-\beta} \\
& =\frac{2}{3 \sqrt{3}} \max _{\|v\|=\|w\|=1} \sum_{\{i, j\} \in E} v_{i} v_{j} w_{i j} .
\end{aligned}
$$

The maximum value of $\sum_{\{i, j\} \in E} v_{i} v_{j} w_{i j}$, whether over $\mathbb{S}^{n-1} \times \mathbb{S}^{m-1}$ or over $\mathbb{S}^{n+m-1}$, can always be attained with $v \geq 0$ and $w \geq 0$, thereby allowing one to take square in (7) and (8). The same proof works for stability number with the replacement of index of summation ' $\{i, j\} \in E$ ' by ' $\{i, j\} \notin E$ '.

\section{Complexity of deciding self-concordance}

The recent resolution of Shor's conjecture by Ahmadi, Olshevsky, Parrilo, and Tsitsiklis [1] shows that deciding the convexity of a quartic polynomial globally over $\mathbb{R}^{n}$ is NP-hard. So the self-concordance of a function that is not a priori known to be convex is NP-hard since deciding whether (1) holds for all $x \in \Omega$ in Definition 1 is already an NP-hard problem. Our complexity result assumes more stringent conditions: (i) Our functions are smooth and convex in $\Omega$ and so (1) is always satisfied and self-concordance reduces to checking (2). (ii) We show that (2) is already NP-hard to check at a single point $x \in \Omega$.

Throughout the following we will require the inputs to our problems to take values in an algebraic number field ${ }^{2}$, e.g., $A \in \mathbb{Q}^{n \times n \times n}, q \in \mathbb{Q}$, to ensure a finite bit-length input. Since an NP-hard problem need not be in the class NP, an NP-hard decision problem can be posed over the reals (e.g. is there an $h \in \mathbb{R}^{n}$ such that $[A(h, h, h)]^{2} \leq q\left[h^{\top} h\right]^{3}$ holds?) without any issue as it is not required to have a polynomial-time checkable certificate.

We will now formulate a decision problem that will lead us to the NP-hardness of selfconcordance. Let $G=(V, E)$ be an undirected graph with $n$ vertices and $m$ edges where $n \geq 2$ and $m \geq 1$. We order the $n$ vertices arbitrarily so that $V=\{1, \ldots, n\}$. We also order the $m$ edges arbitrarily so that

$$
E=\left\{\left\{i_{k}, j_{k}\right\}: k=1, \ldots, m\right\}
$$

2 We will only encounter simple quadratic and cubic extensions of $\mathbb{Q}$, see (18) and (11). For $q \in \mathbb{Q}$, elements of $\mathbb{Q}(\sqrt{q})$ and $\mathbb{Q}(\sqrt[3]{q})$ may be written as $a+b \sqrt{q}$ and $a+b \sqrt[3]{q}+c(\sqrt[3]{q})^{2}$ respectively with $a, b, c \in \mathbb{Q}$, thus representable by pairs and triples of rational numbers. 
Define $B_{G}=\left[b_{i j k}\right]_{i, j, k=1}^{n+m} \in \mathbb{Q}^{(n+m) \times(n+m) \times(n+m)}$ by

$$
b_{i j k}= \begin{cases}1 & \text { if } i=i_{k} \in V, j=j_{k} \in V,\left\{i_{k}, j_{k}\right\} \in E, \\ 0 & \text { otherwise. }\end{cases}
$$

$B_{G}$ is not a symmetric hypermatrix. Let $A_{G}=\left[a_{i j k}\right]_{i, j, k=1}^{n+m} \in \mathbb{Q}^{(n+m) \times(n+m) \times(n+m)}$ be the symmetrization of $B_{G}$, i.e.,

$$
a_{i j k}=\frac{1}{3 !}\left(b_{i j k}+b_{i k j}+b_{j i k}+b_{j k i}+b_{k i j}+b_{k j i}\right)
$$

for all $i, j, k \in\{1, \ldots, n+m\}$. So $A_{G}$ is symmetric, i.e., $a_{i j k}=a_{i k j}=a_{j i k}=a_{j k i}=$ $a_{k i j}=a_{k j i}$, and furthermore $A_{G}(h, h, h)=B_{G}(h, h, h)$. Let us denote the coordinates of $h \in \mathbb{R}^{n+m}$ by

$$
h=\left(v_{1}, \ldots, v_{n}, w_{i_{1} j_{1}}, \ldots, w_{i_{m} j_{m}}\right) .
$$

In which case,

$$
\begin{aligned}
A_{G}(h, h, h) & =B_{G}(h, h, h)=\sum_{i, j, k=1}^{m+n} b_{i j k} h_{i} h_{j} h_{k} \\
& =\sum_{k=1}^{m} v_{i_{k}} v_{j_{k}} w_{i_{k} j_{k}}=\sum_{\{i, j\} \in E} v_{i} v_{j} w_{i j} .
\end{aligned}
$$

By Theorem 2,

$$
\max _{h \neq 0}\left[\frac{A_{G}(h, h, h)}{\|h\|^{3}}\right]^{2}=\max _{\|h\|=1}\left[A_{G}(h, h, h)\right]^{2}=\frac{2}{27}\left(1-\frac{1}{\omega(G)}\right) .
$$

The CLIQUE problem asks if for a given graph $G$ and a given $k \in \mathbb{N}$, whether $G$ has a clique of size $k$. CLIQUE is well-known to be NP-complete [9]. So deciding if $\omega(G) \geq k$, or equivalently, $\omega(G)>k-1$, is an NP-complete problem; by (10), so is deciding if

$$
\max _{h \neq 0}\left[\frac{A_{G}(h, h, h)}{\|h\|^{3}}\right]^{2}>\frac{2}{27}\left(1-\frac{1}{k-1}\right) .
$$

Hence deciding if there exists an $h \in \mathbb{R}^{n+m}$ for which

$$
\left[A_{G}(h, h, h)\right]^{2}>\frac{2}{27}\left(1-\frac{1}{k-1}\right)\left[h^{\top} h\right]^{3}
$$

is an NP-hard problem. As $q=\frac{2}{27}\left[1-(k-1)^{-1}\right] \in \mathbb{Q}$, this problem is of the form:

Problem 1 Given a symmetric $A \in \mathbb{Q}^{(n+m) \times(n+m) \times(n+m)}$ and a positive $q \in \mathbb{Q}$, does there exists an $h \in \mathbb{R}^{n+m}$ for which $[A(h, h, h)]^{2}>q\left[h^{\top} h\right]^{3}$ ?

Let $\sigma \in \mathbb{Q}, \sigma>0$, be a self-concordance parameter and let

$$
\gamma:=\frac{1}{3}\left[\frac{1}{2 \sigma}\left(1-\frac{1}{k-1}\right)\right]^{1 / 3} .
$$

We follow the notation in Sect. 2. Let $\Omega$ be the $\varepsilon$-ball $B_{\varepsilon}(0)=\left\{x \in \mathbb{R}^{n+m}:\|x\|<\varepsilon\right\}$ where $\varepsilon>0$ is to be chosen later. We are interested in deciding self-concordance at $x=0$ of the cubic polynomial $f: \Omega \rightarrow \mathbb{R}$ defined by

$$
f(x)=\frac{\gamma}{2} x^{\top} x+A_{G}(x, x, x)=\frac{\gamma}{2} \sum_{i=1}^{n+m} x_{i}^{2}+\sum_{i, j, k=1}^{n+m} a_{i j k} x_{i} x_{j} x_{k} .
$$


We have $\nabla^{2} f(0)=\gamma I$ where $I$ is the $(n+m) \times(n+m)$ identity matrix. Since $\gamma>0, \nabla^{2} f(x)$ is strictly positive definite in a neighborhood of $x=0$ and so there is some $B_{\varepsilon}(0)$ on which $f$ is convex, giving us our $\varepsilon$. Also, $\nabla^{3} f(0)=A_{G}$.

Hence $\nabla^{2} f(0)(h, h)=\gamma h^{\top} h=\gamma\|h\|_{2}^{2}, \nabla^{3} f(0)(h, h, h)=A_{G}(h, h, h)$, and $f$ is selfconcordant at the origin with parameter $\sigma \in \mathbb{Q}$ if and only if

$$
\left[A_{G}(h, h, h)\right]^{2} \leq 4 \sigma \gamma^{3}\left[h^{\top} h\right]^{3}=\frac{2}{27}\left(1-\frac{1}{k-1}\right)\left[h^{\top} h\right]^{3}
$$

for all $h \in \mathbb{R}^{n+m}$. This problem is of the form:

Problem 2 Given a symmetric $A \in \mathbb{Q}^{(n+m) \times(n+m) \times(n+m)}$ and a positive $q \in \mathbb{Q}$, is it true that for every $h \in \mathbb{R}^{n+m}$, we have $[A(h, h, h)]^{2} \leq q\left[h^{\top} h\right]^{3}$ ?

Problems 1 and 2 are mathematically equivalent, being logical complements of each other. However they may or may not have the same computational complexity depending on our choice of reduction [16]. Using Cook reduction, also know as polynomial-time Turing reduction [16], Problems 1 and 2 have equivalent computational complexity, i.e., deciding self-concordance is NP-hard. However, using Karp reduction, also know as polynomialtime many-one reduction [16], the NP-hardness of Problem 1 implies the co-NP-hardness of Problem 2. In either case, our conclusion is that self-concordance is intractable.

Theorem 3 Deciding whether a cubic polynomial is self-concordant at the origin is NP-hard under Cook reduction and co-NP-hard under Karp reduction.

Deciding self-concordance on the whole of $\Omega$ is of course at least as hard as deciding selfconcordance at a point in $\Omega$ and we obtain the following.

Corollary 1 For any $\Omega$ and any $\sigma>0$, membership in $S_{\sigma}(\Omega)$ is NP-hard.

The argument in this section clearly works not just for cubic polynomials but for any $f \in C^{3}(\Omega)$ as long as $0 \in \Omega, \nabla^{2} f(0)=\gamma I$, and $\nabla^{3} f(0)=A_{G}$ - other derivatives and the remainder term in the Taylor expansion of $f$ at $x=0$ may be chosen arbitrarily as long as $f$ stays convex in $\Omega$. This flexibility allows one to extend the construction above to functions with other desired properties. For instance, we may want an example where $\Omega=\mathbb{R}^{n}$ and since cubic polynomials cannot be convex on the whole of $\mathbb{R}^{n}$, we will need a quartic $f$ and therefore need to choose $\nabla^{4} f(0)$ accordingly; or we may want an example where $f$ is a barrier function, which is equivalent to $f$ having an epigraph $\left\{(x, t) \in \mathbb{R}^{n+1}: x \in \Omega, f(x) \leq t\right\}$ that is closed. One may trivially replace 0 by any point $a \in \mathbb{R}^{n}$ by considering the function $f_{a}(x)=f(x-a)$ on $\Omega=B_{\varepsilon}(a)$.

While we have proved our hardness result for functions on $\Omega \subseteq \mathbb{R}^{n}$, it is easy to extend this to any $\mathbb{R}$-vector space, for example, symmetric matrices $\mathbb{S}^{n \times n}$ or polynomials $\mathbb{R}\left[x_{1}, \ldots, x_{n}\right]$, or even Riemannian manifolds with a non-trivial class of geodesically convex functions (i.e., not just the constant functions). Since self-concordance at a point is a local property, a choice of coordinate patch would transform the problem to one in $\mathbb{R}^{n}$; and by our remark at the end of Sect. 2, it will in fact be independent of our choice of coordinates.

The reason our conclusion in Theorem 3 is given as NP- and co-NP-hardness as opposed to NP- and co-NP-completeness is that checking a condition like $[A(h, h, h)]^{2} \leq q\left[h^{\top} h\right]^{3}$, even if the certificate $h$ is in $\mathbb{Q}^{n}$, could well require exponential time when time complexity is measured in units of bit operations. 


\section{Inapproximability of optimal self-concordance parameter}

Let $A \in \mathbb{Q}^{n \times n \times n}$ be symmetric and $f: \Omega \rightarrow \mathbb{R}$ be defined by the cubic polynomial $f(x)=\frac{1}{2} x^{\top} x+A(x, x, x)$. As in Sect. $4, \Omega$ is chosen to be a neighborhood of the origin so that $f$ is convex on $\Omega$. The condition (2) for self-concordance of $f$ at $x=0$ with parameter $\sigma>0$ may be written as

$$
|A(h, h, h)| \leq 2 \sqrt{\sigma}\|h\|_{2}^{3}
$$

for all $h \in \mathbb{R}^{n}$. This is equivalent to requiring

$$
\max _{h \neq 0} \frac{A(h, h, h)}{\|h\|_{2}^{3}} \leq 2 \sqrt{\sigma}
$$

as $A(-h,-h,-h)=-A(h, h, h)$ and we may drop the absolute value in (12).

Since $A \in \mathbb{R}^{n \times n \times n}$ is a symmetric 3-hypermatrix, the spectral norm [6] of $A$ may be expressed as follows:

$$
\|A\|_{2,2,2}:=\max _{h_{1}, h_{2}, h_{3} \neq 0} \frac{A\left(h_{1}, h_{2}, h_{3}\right)}{\left\|h_{1}\right\|_{2}\left\|h_{2}\right\|_{2}\left\|h_{3}\right\|_{2}}=\max _{h \neq 0} \frac{A(h, h, h)}{\|h\|_{2}^{3}},
$$

where the second equality follows from Banach's result on the polarization constant of Hilbert spaces (see [2] and [17]). Hence the optimal self-concordance parameter of $f$ at $x=0$, i.e., the smallest value of $\sigma$ so that (13) holds, is given by

$$
\sigma_{\mathrm{opt}}=\frac{1}{4}\|A\|_{2,2,2}^{2} .
$$

The spectral norm of a 3-hypermatrix is NP-hard to approximate to within a certain constant factor by [6, Theorem 1.11], which we state here for easy reference.

Theorem 4 (Hillar-Lim) Let $A \in \mathbb{Q}^{n \times n \times n}$ and $N$ be the input size of $A$ in bits. Then it is $N P$-hard to approximate $\|A\|_{2,2,2}$ to within a factor of $1-\varepsilon$ where

$$
\varepsilon=1-\left(1+\frac{1}{N(N-1)}\right)^{-1 / 2}=\frac{1}{2 N(N-1)}+O\left(\frac{1}{N^{4}}\right) .
$$

By (14) and Theorem 4, $\sigma_{\text {opt }}$ is NP-hard to approximate to within a factor of $\frac{1}{4}(1-\varepsilon)^{2}$ and consequently we have the following inapproximability result.

Corollary 2 There is no polynomial time approximation scheme for determining the optimal self-concordance parameter $\sigma_{\mathrm{opt}}$ unless $P=N P$.

See [5] and [7] for more extensive approximability results and approximation algorithms (that are not PTAS). The results in [7] for quartic polynomials would apply to the optimal second-order self-concordance parameter in the next section.

\section{Complexity of deciding second-order self-concordance}

There is also an interesting notion of second-order self-concordance due to Jarre [10]. This requires that $f \in C^{4}(\Omega)$ and is given by a condition involving the matrix $\nabla^{2} f(x) \in \mathbb{R}^{n \times n}$ and the 4-hypermatrix $\nabla^{4} f(x) \in \mathbb{R}^{n \times n \times n \times n}$. 
Definition 2 (Jarre) If $\Omega \subseteq \mathbb{R}^{n}$ is a convex open set, then $f: \Omega \rightarrow \mathbb{R}$ is said to be second-order self-concordant with parameter $\tau>0$ at $x \in \Omega$ if

$$
\nabla^{2} f(x)(h, h) \geq 0
$$

and

$$
\nabla^{4} f(x)(h, h, h, h) \leq 6 \tau\left[\nabla^{2} f(x)(h, h)\right]^{2}
$$

for all $h \in \mathbb{R}^{n} ; f$ is second-order self-concordant on $\Omega$ if it is so for all $x \in \Omega$.

Note that

$$
\nabla^{4} f(x)(h, h, h, h)=\sum_{i, j, k, l=1}^{n} \frac{\partial^{4} f(x)}{\partial x_{i} \partial x_{j} \partial x_{k} \partial x_{l}} h_{i} h_{j} h_{k} h_{l},
$$

is a quartic polynomial in $h$ for any fixed $x \in \Omega$.

We follow the same argument in Sect. 4 to show that deciding (16) is NP-hard. This time the result would be deduced from Motzkin-Straus Theorem except that for better parallelism with Sect. 4, we will use the quartic-maximization-over-sphere form (6) instead of the quadraticmaximization-over-simplex form (3).

Given a graph $G=(V, E)$ with $n$ vertices and $m$ edges where $n \geq 2$ and $m \geq 1$, we define $B_{G} \in \mathbb{Q}^{n \times n \times n \times n}$ by

$$
b_{i j k l}= \begin{cases}1 & i=k, j=l, \text { and }\{i, j\} \in E, \\ 0 & \text { otherwise. }\end{cases}
$$

Let $A_{G}=\left[a_{i j k l}\right] \in \mathbb{Q}^{n \times n \times n \times n}$ be the symmetrization of $B_{G}$, i.e.,

$$
a_{i j k l}=\frac{1}{4 !}\left(b_{i j k l}+b_{i j l k}+\cdots+b_{l k j i}\right)
$$

where the indices run over all 24 possible permutations. So $A=\left[a_{i j k l}\right]_{i, j, k, l=1}^{n} \in \mathbb{Q}^{n \times n \times n \times n}$ is symmetric and $A_{G}(h, h, h, h)=B_{G}(h, h, h, h)$. As in (6),

$$
\max _{\|h\|=1} A_{G}(h, h, h, h)=\max _{h \in \mathbb{S}^{n-1}} \sum_{\{i, j\} \in E} h_{i}^{2} h_{j}^{2}=\frac{1}{2}\left(1-\frac{1}{\omega(G)}\right)
$$

by Motzkin-Straus Theorem. As in Sect. 4 , for $k \geq 2$, deciding if a $k$-clique exists in $G$ is equivalent to deciding if $\omega(G)>k-1$. So deciding if

$$
A_{G}(h, h, h, h)>\frac{1}{2}\left(1-\frac{1}{k-1}\right)\left[h^{\top} h\right]^{2}
$$

for some $h \in \mathbb{R}^{n}$ is NP-hard. Given self-concordance parameter $\tau \in \mathbb{Q}, \tau>0$, let

$$
\gamma:=\left[\frac{1}{12 \tau}\left(1-\frac{1}{k-1}\right)\right]^{1 / 2} .
$$

We may now define $f: \Omega \rightarrow \mathbb{R}$ accordingly as the quartic polynomial

$$
f(x)=\frac{\gamma}{2} x^{\top} x+A_{G}(x, x, x, x)=\frac{\gamma}{2} \sum_{i=1}^{n} x_{i}^{2}+\sum_{i, j, k, l=1}^{n} a_{i j k l} x_{i} x_{j} x_{k} x_{l} .
$$

Hence $\nabla^{2} f(0)(h, h)=\gamma h^{\top} h=\gamma\|h\|_{2}^{2}$ and $\nabla^{4} f(0)(h, h, h, h)=A_{G}(h, h, h, h)$. Again we choose $\Omega$ to be a neighborhood of the origin so that $f$ is convex on $\Omega$. So $f$ is second-order self-concordant at $x=0$ with parameter $\tau$ if and only if

$$
A_{G}(h, h, h, h) \leq 6 \tau \gamma^{2}\left[h^{\top} h\right]^{2}=\frac{1}{2}\left(1-\frac{1}{k-1}\right)\left[h^{\top} h\right]^{2}
$$


is satisfied for all $h \in \mathbb{R}^{n}$. Since the problem of deciding if there exists an $h \in \mathbb{R}^{n}$ satisfying (17) and the problem of deciding if (19) is satisfied for all $h \in \mathbb{R}^{n}$ are logical complements and the former is NP-hard, we have the following.

Theorem 5 Deciding if a quartic polynomial is second-order self-concordant at the origin is NP-hard under Cook reduction and co-NP-hard under Karp reduction.

Self-concordance and second-order self-concordance are conditions involving high-order tensors (orders 3 and 4 respectively), their NP-hardness serves as yet another reminder of the complexity of tensor problems [6].

Acknowledgements We would like to thank the anonymous referees for their careful reading and helpful suggestions.

\section{References}

1. Ahmadi, A.A., Olshevsky, A., Parrilo, P.A., Tsitsiklis, J.N.: NP-hardness of deciding convexity of quartic polynomials and related problems. Math. Progr. Ser. A 137(1-2), 453-476 (2013)

2. Banach, S.: Über homogene polynome in $\left(L^{2}\right)$. Stud. Math. 7(1), 36-44 (1938)

3. Boyd, S., Vandenberghe, L.: Convex Optimization. Cambridge University Press, Cambridge (2004)

4. De Klerk, E.: The complexity of optimizing over a simplex, hypercube or sphere: a short survey. Cent. Eur. J. Oper. Res. 16(2), 111-125 (2008)

5. He, S., Li, Z., Zhang, S.: Approximation algorithms for homogeneous polynomial optimization with quadratic constraints. Math. Progr. Ser. B 125(2), 353-383 (2010)

6. Hillar, C. J., Lim, L.-H.: Most tensor problems are NP-hard. J. ACM 60(6), 39 (Art. 45) (2013)

7. Hou, K., So, A.M.-C.: Hardness and approximation results for $L_{p}$-ball constrained homogeneous polynomial optimization problems. Math. Oper. Res. 39(4), 1084-1108 (2014)

8. Jiang, B., Li, Z., Zhang, S.: On cones of nonnegative quartic forms. Found. Comput. Math. (2016). doi:10. 1007/s10208-015-9286-4

9. Karp, R.M.: Reducibility among combinatorial problems. In: Miller, R.E., Thatcher, J.W. (eds.) Complexity of Computer Computations, pp. 85-103. Plenum, New York (1972)

10. Jarre, F.: A new line-search step based on the Weierstrass $\wp$-function for minimizing a class of logarithmic barrier functions. Numer. Math. 68(1), 81-94 (1994)

11. Lang, S.: Differential and Riemannian Manifolds, 3rd edn., Graduate Texts in Mathematics, vol. 160. Springer, New York (1995)

12. Lim, L.-H.: Tensors and hypermatrices. In: Hogben, L. (ed.) Handbook of Linear Algebra, 2nd edn. CRC Press, Boca Raton (2013)

13. Motzkin, T., Straus, E.G.: Maxima for graphs and a new proof of a theorem of Turán. Can. J. Math. 17, 533-540 (1965)

14. Nesterov, Yu.: Random walk in a simplex and quadratic optimization over convex polytopes. Preprint (2003). http://edoc.bib.ucl.ac.be:83/archive/00000238/01/dp2003-71

15. Nesterov, Yu., Nemirovskii, A.: Interior-Point Polynomial Algorithms in Convex Programming. SIAM Studies in Applied Mathematics, vol. 13. SIAM, Philadelphia (1994)

16. Papadimitriou, C.H.: Computational Complexity. Addison-Wesley, Reading (1994)

17. Pappas, A., Sarantopoulos, Y., Tonge, A.: Norm attaining polynomials. Bull. Lond. Math. Soc. 39(2), 255-264 (2007) 